\title{
Brief Report \\ Immersive Virtual Reality Exergame Promotes the Practice of Physical Activity in Older People: An Opportunity during COVID-19
}

\author{
Pablo Campo-Prieto ${ }^{1, *(D)}$, Gustavo Rodríguez-Fuentes ${ }^{1}$ (D) and José $\mathbf{M}^{\text {a }}$ Cancela-Carral ${ }^{2}$ (D) \\ 1 HealthyFit Research Group, Department of Functional Biology and Health Sciences, Faculty of Physiotherapy, \\ Galicia Sur Health Research Institute (IIS Galicia Sur), University of Vigo, E-36005 Pontevedra, Spain; \\ gfuentes@uvigo.es \\ 2 HealthyFit Research Group, Department of Special Didactics, Faculty of Education and Sports Science, \\ Galicia Sur Health Research Institute (IIS Galicia Sur), University of Vigo, E-36005 Pontevedra, Spain; \\ chemacc@uvigo.es \\ * Correspondence: pcampo@uvigo.es; Tel.: +34-9868-01700
}

check for updates

Citation: Campo-Prieto, P.; Rodríguez-Fuentes, G.; Cancela-Carral, J.M. Immersive Virtual Reality Exergame Promotes the Practice of Physical Activity in Older People: An Opportunity during COVID-19. Multimodal Technol. Interact. 2021, 5, 52. https://doi.org/ $10.3390 / \mathrm{mti} 5090052$

Academic Editor: Mark Billinghurst

Received: 5 August 2021

Accepted: 25 August 2021

Published: 1 September 2021

Publisher's Note: MDPI stays neutral with regard to jurisdictional claims in published maps and institutional affiliations.

Copyright: (C) 2021 by the authors Licensee MDPI, Basel, Switzerland. This article is an open access article distributed under the terms and conditions of the Creative Commons Attribution (CC BY) license (https:// creativecommons.org/licenses/by/ $4.0 /)$.

\begin{abstract}
Life expectancy has increased in recent years. Physical activity has been postulated as a key element in active aging strategies. However, adherence to physical exercise programs has traditionally been low among the elderly, and the current situation of the COVID-19 pandemic has added extra impediments. Immersive virtual reality (IVR) devices could motivate this population to practice exercise. This study aimed to analyse the use of IVR exergames as a tool to facilitate physical exercise in older people. Four healthy older adults (males, 65-77 years) participated in the study. They carried out two exergaming sessions with HTC Vive Pro ${ }^{\text {TM }}$. Outcomes were evaluated using the Simulator Sickness Questionnaire (SSQ), System Usability Scale (SUS), Game Experience Questionnaire (GEQ post-game module), an ad hoc satisfaction questionnaire, and perceived effort. All participants completed the sessions without adverse effects, with no SSQ symptoms reported. SUS scores were high in both sessions (SUS > 85/100). Post-game GEQ scores were 3.08-3.41/4 (positive experiences) and 0.08-0.16/4 (negative experiences). Opinions showed high levels of satisfaction with the experience. Exergaming programs, based on commercial head-mounted displays, are a feasible alternative to traditional senior exercise, and could be a solution to the current situation that has arisen from the impact of the COVID-19 pandemic.
\end{abstract}

Keywords: virtual reality; older adults; video games; healthy aging; exergaming; health promotion; games for health; physical therapy modalities

\section{Introduction}

Life expectancy has increased in recent years and, as a result, subjects relating to older people in our society have become especially relevant [1]. In this new reality, active aging policies seem to be important [2] in the desire to keep the senior population healthy, active, and autonomous for as long as possible [3], thus avoiding their functional decline [4].

One of the cornerstones of active aging is the practice of physical activity, the main objective of which is to maintain or improve the individual's functionality. Firstly, exercise combats sedentary lifestyle but it also contributes to increased feelings of personal achievement, competence, and self-efficacy, as well as having a positive impact socially [5].

There are numerous scientific studies showing that physical exercise programs play an essential role not only in slowing down the degenerative process, but also in improving the individual's conditional and coordination capacities, having a positive impact on their functional independence and, consequently, on their quality of life [6,7].

Adherence to programs based on the practice of physical exercise and sports has traditionally been low across this age group [8]. 
Furthermore, systematic reviews indicate that the proportion of older adults who complete group exercise programs ranges from 65 to $86 \%$, attendance rates at sessions fluctuate between 58 and $77 \%$, and the average number of exercise sessions completed at home is between 1.5 and 3 days per week $[9,10]$.

An added and unexpected difficulty has been the situation caused by the outbreak of coronavirus disease 2019 (COVID-19), caused by SARS-CoV-2. The current pandemic has caused unprecedented disruption to all aspects of our lives worldwide [11]. Governments have enacted numerous measures to prevent the spread of the virus, including mass lockdowns, which have reduced social activities to an absolute minimum [12], including sports and leisure activities. The wearing of masks in all public places is another measure that has been widely adopted [13]. Recent studies have found that the practice of physical activity whilst wearing a mask increases the physiological load of the body, raising the heart rate and perceived effort. This fact is one that should be especially considered in more vulnerable, older, or comorbid populations [14].

The COVID-19 pandemic is bringing about a decrease in physical activity. Mutz and Gerke [15] analysed this situation in Germany-which has not had a lockdown as restrictive as the one imposed in Spain-and although they found that $55.8 \%$ of those over 65 years of age were considered active, $53 \%$ of these had reduced their physical activity.

To alleviate this exercise deficit, different initiatives have emerged that encourage the practice of physical exercise, seeking to motivate individuals to engage in physical activity and maintain adherence to exercise, in addition to preventing COVID-19 infection [16]. Adherence to exercise programmes is fundamental to achieving and maintaining the health benefits that they provide. Active video games (exergames), originally created for entertainment, are a potentially fun and motivating alternative for exercising at home [17].

There are examples of the use of exergames across different population groups, including older people. Subjects have played different games and activities on the Nintendo Wii [18] or Xbox Kinect [19] platforms, and the physiological effects of different activities have been evaluated after exergaming. Other studies have also proven the capability of exergames to improve physical activity levels, the performance of daily activities, balance, and cognitive functions in older people [20].

A further step would be to use the innovative head-mounted displays (HMDs) and immersive virtual reality (IVR), with which users can try out completely immersive experiences in three-dimensional environments on demand-experiences that are pleasant, and motivate users to engage in physical exercise [21,22]. This exercise can be carried out conveniently, without having to travel to sports facilities, and can also offer the possibility of participating in group activities, but without social contact. Some structural problems (access to technology, the need for initial training, or cybersickness) have limited the application of this VR technology in the most vulnerable groups, such as the senior population [23].

However, our previous work reported that the use of this technology by older people is lacking [24], in most cases have addressed the use of assessment or rehabilitation tools in people with pathologies such as Parkinson's disease [25] or post-stroke symptoms [26]. This study aimed to analyse the use of IVR exergames using a commercial HMD, as a tool to facilitate physical exercise in healthy older people.

\section{Materials and Methods}

\subsection{Participants}

Four healthy older adults participated in the study (Table 1). After being invited to participate in the IVR physical activity test, they completed a brief questionnaire about their physical health, previous experience with video games, frequency of physical activity, and absolute or relative contraindications for exercise. Subjects' exclusion criteria were the inability to correctly respond to the assessment protocol according to the clinician 's judgment; the presence of cardiovascular, pulmonary, or musculoskeletal conditions that, according to the physiotherapist's judgment, affect patients' ability to participate in the 
study; or the presence of severe visual loss that could interfere with the ability to see the IVR simulation, as well as vertigo, epilepsy, or psychosis. After the objectives and procedures had been explained, informed consent was obtained.

Table 1. Demographic characteristics of the participants.

\begin{tabular}{ccccc}
\hline Characteristics & P1 & P2 & P3 & P4 \\
\hline Age (years) & 77 & 67 & 65 & 74 \\
Gender & Male & Male & Male & Male \\
BMI $\left(\mathrm{kg} / \mathrm{m}^{2}\right)$ & 24.09 & 30.07 & 26.19 & 24.80 \\
Gaming experience & No & No & No & No \\
Exercise habits & AE $(3 \mathrm{~d} / \mathrm{w})$ & Sedentary & Sedentary & Sedentary \\
\hline
\end{tabular}

AE: aerobic exercise; BMI: body mass index; $\mathrm{d} / \mathrm{w}$ : days per week.

\subsection{Experimental Setup}

This study was carried out in the Healthy Physical Activity Lab (Faculty of Education and Sports Sciences, University of Vigo, Pontevedra, Spain). The immersive virtual environment was created using the HTC Vive Pro ${ }^{\text {TM }}$ (HTC Corporation, Taoyuan City, Taiwan) commercial entertainment device. This system consists of a head-mounted display, two wireless hand controllers, two external sensors to delimit the gaming surface, a wireless adapter, and the Viveport support software (https:/ / viveport.com accessed on 15 July 2021), supported by a desktop computer (CPU: Intel Core I7 7700 at $3.6 \mathrm{GHz}, 1 \mathrm{~Tb}$ HDD Sata 3.5 and NVIDIA GeForce RTX 2070 GPUs). A led tv screen was used to guide activities and set up technical aspects of the device. A $5 \mathrm{~m}^{2}$ play area was defined following the manufacturer's installation recommendations and taking into consideration the dimensions of the area selected for the study.

Two interventions were set on two different days, and participants used IVR for approximately 10-12 min. The researchers selected one experience (introduction) and two exergames (warm-up and training). Participants were instructed to stop the session if they experienced discomfort or excessive fatigue. In view of the characteristics of the participants, the playing position chosen was standing. Heart rate (HR) was monitored and, at the end of the activity, in a sitting position, the participants were asked if they had experienced discomfort linked to cybersickness. Each participant had an individual session guided and supervised by a physiotherapist with experience with older people (Figure 1).

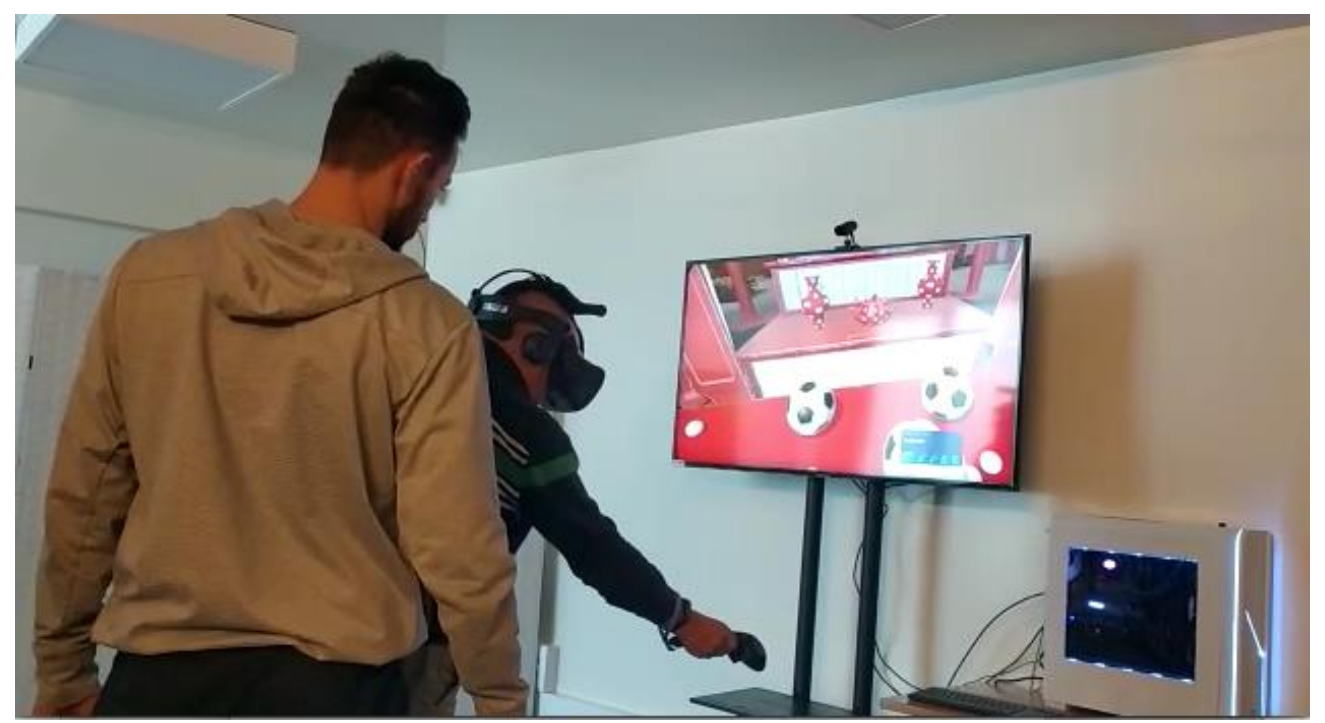

Figure 1. Participant during an individual session, guided and supervised by a physiotherapist. 


\subsection{Related Work}

\subsubsection{Acclimatization}

Since none of the participants had any previous gaming experience, an intermediate activity (acclimatization) was planned to introduce them to the immersive environment. This activity was organized in order to introduce the exergaming sessions: an introduction talk and an explanation of how to handle the device, both by demonstration and by allowing the participants to try out the trackers and the HMD. Steam VR Home (available in the library of https: / /viveport.com, accessed on 15 July 2021), which simulates being in a room with a door opening onto an exterior mountain landscape, served as a test scenario (Figure 2) (duration: $\sim 8 \mathrm{~min}$ ). During this time, they answered questions that the therapists asked about the environment, in order to test their presence and immersion in the game, as well as performing body movements (real and virtual). This previous experience was led by health and exercise professionals, who chose to provide a first contact with the IVR in a nice, fun, and quiet virtual scenario to ensure good acceptability.

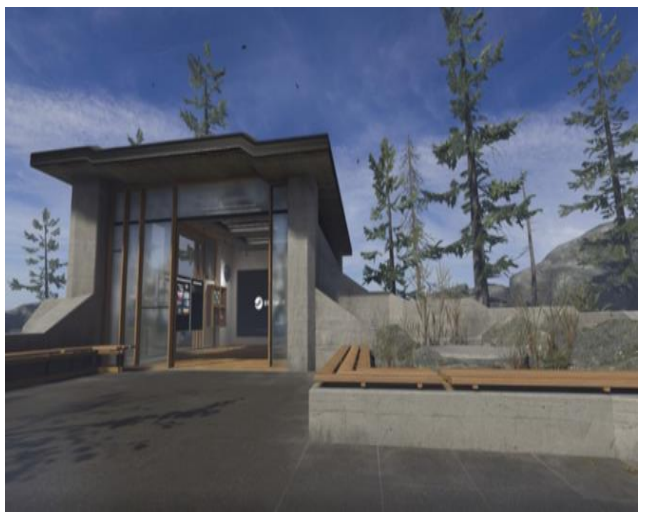

(a)

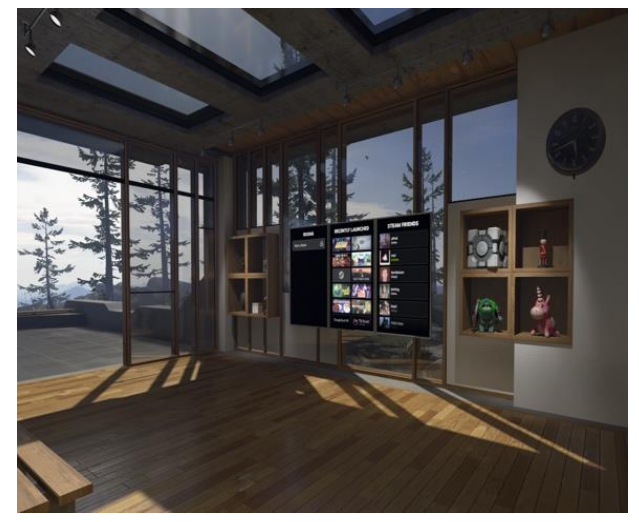

(b)

Figure 2. Screenshots of the introduction session (Steam VR Home): (a) room placed in a mountain landscape; (b) inside room with a door opening onto an exterior place with some objects to manipulate.

\subsubsection{Exergaming Session 1}

For the first training, BOX VR (available in the library of https:/ / viveport.com, accessed on 15 July 2021), which simulates being in a gym with a boxing theme, was selected. The continuous presentation of different stimuli requires coordinated movements in time and space of the upper limbs, trunk, and head, but also of the lower limbs, thus varying the initial static standing position with squats and lateral movements. The music aids the performance, and was selected for its multicomponent function (aerobic, balance, strength, coordination, and agility), highly recommended in the target population and supported by four physical therapists from our lab (duration: $\sim 6 \mathrm{~min}$ ).

Since the initial experience was feasible, safe, and the participants reported a high level of satisfaction, it was decided to schedule a new session two weeks later. Furthermore, in view of the previous positive experience, the acclimatization test was eliminated and replaced with a warm-up activity.

\subsubsection{Exergaming Session 2}

This session began with a warm-up activity using the NVIDIA VR Fun House experience (available in the library of https:/ / www.steamvr.com/en/, accessed on 15 July 2021), where users find themselves at a carnival scenario where they can take an active part in the experience by playing minigames with different activities, such as throwing objects, archery, handling a sword, etc. (Figure 3). These activities require repetitive movements in all planes, and could be considered a suitable warm-up prior to a training session. As in the previous sessions, control measures included supervision by the physiotherapist, answers about discomfort, and HR monitoring (duration: $~ 5 \mathrm{~min}$ ). 


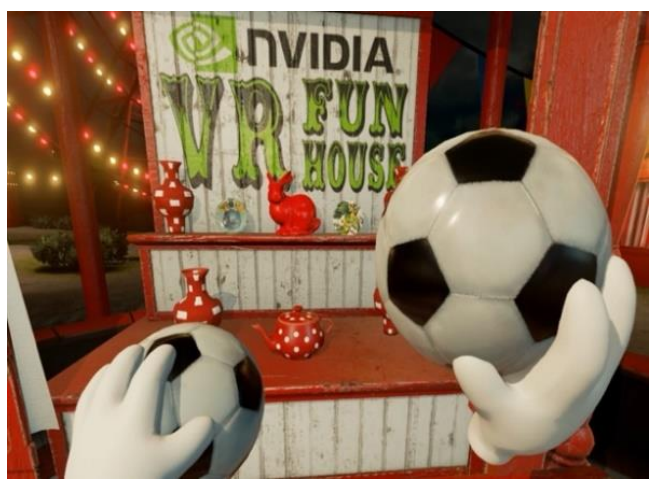

(a)

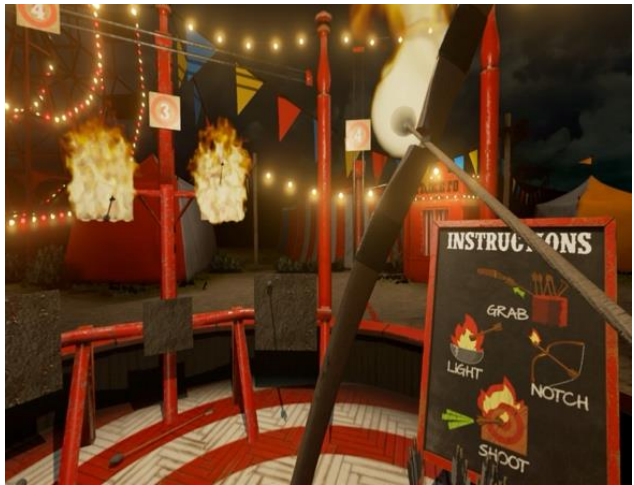

(b)

Figure 3. Screenshots of the warm-up session (NVIDIA VR Fun House): (a) participant throws balls to break objects; (b) participant practices archery and shoots at different targets.

For the training, the same virtual experience as session 1 (BOX VR) was used (Figure 4), for the same length of time, and with the same control and safety measures.

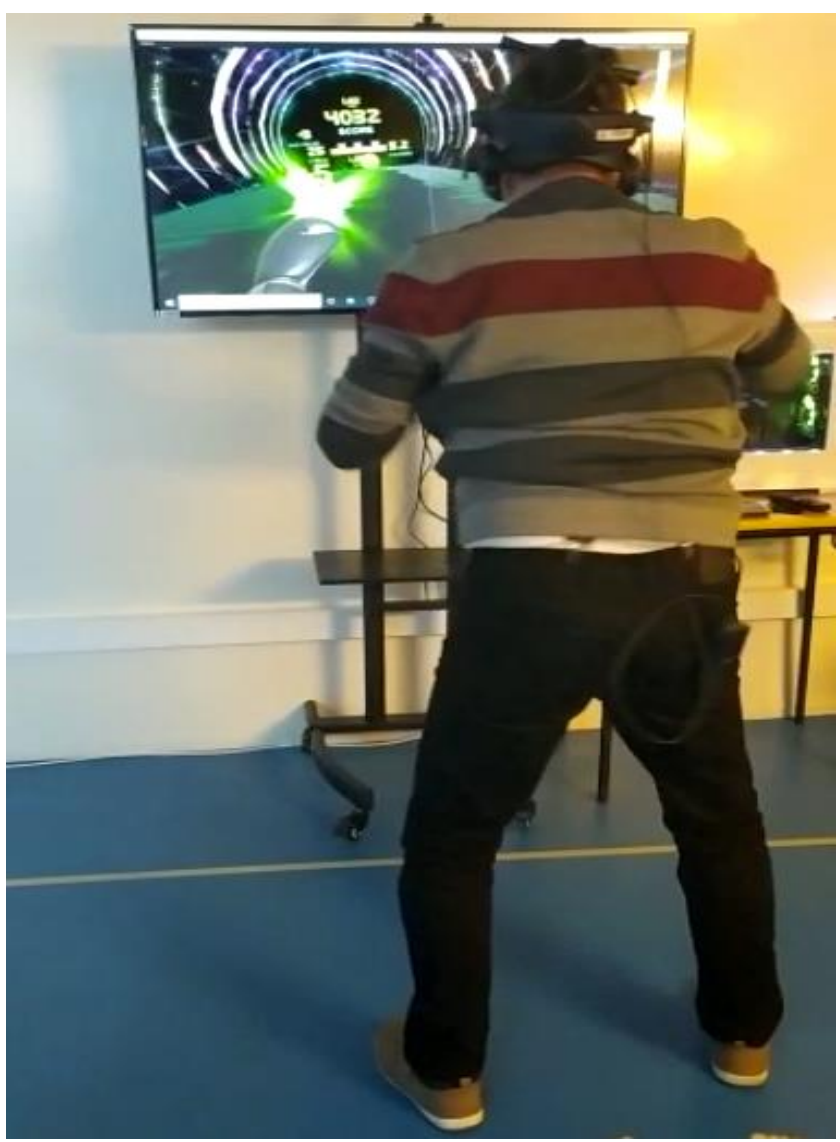

Figure 4. Participant during boxing exergame session (BOX VR).

\subsection{Assessment Tools}

All participants completed the following questionnaires:

- Safety of the immersive experience through the Simulator Sickness Questionnaire (SSQ) [27]. This questionnaire, originally designed to be applied in simulators, consists of 16 items, grouped into 3 subscales and divided by symptomatology. Each item is assessed on a four-point scale ( $0=\mathrm{I}$ feel nothing, $1=\mathrm{a}$ little, $2=$ medium, and $3=\mathrm{a}$ lot), and the total score results from the sum of the scores of the three subscales. This 
tool has been widely used to measure the frequency of cybersickness in the general population $[28,29]$.

- Usability of the proposed system through the System Usability Scale (SUS) [30]. The SUS was developed as a survey that allows professionals to evaluate the usability of a product/service in a quick and easy manner. The SUS is a Likert-type scale that includes 10 questions. Participants rate each question from 1 to 5 according to their degree of agreement or disagreement with what they are reading, where 5 means that they completely agree and 1 means that they completely disagree. The resulting algorithm determines a score out of a maximum of 100 points [31].

- Opinions using the Game Experience Questionnaire (post-game GEQ) module [32]. The GEQ is a questionnaire that consists of three modules (main module, social module, and post-game module). Modules 1 and 2 investigate the player's feelings and thoughts, while module 3 assesses how players felt after they stopped playing. This last module, as used in our study, is a Likert-type scale consisting of 17 items, where the responses are rated according to the intensity of the feelings (where 0 is nothing and 4 is extreme). These items are framed over 4 components that are scored individually, and whose average results in a maximum score of 4, encompassing the following: positive experiences, negative experiences, tiredness, and return to reality [33].

- An ad hoc satisfaction questionnaire consisting of 5 questions was developed to identify the strengths and weaknesses of the intervention. The questionnaire was the same as one used in a previous study [22], and includes the following questions: "How was the experience?"; "Would you repeat the experience with IVR"; "Would you recommend the experience of IVR?"; "Do you think the exercise is suitable for people of your age? Why?"; and free comments.

- $\quad$ Effort scores (perceived effort and HR), using the Borg perceived effort scale [34], and by monitoring the average HR with a Mi Smart Band 4 wristband and the Mi Fit version 4.0.14 app (Xiaomi, Haidian, Pekin).

These assessment tools have been previously used in similar research [25,35], and they are intended to evaluate the feasibility and applicability of physical exercise/sports activities based around completely immersive VR environments for an elderly population.

\section{Results}

All participants completed the training sessions successfully, without any adverse effects, in both sessions. Table 2 shows effort scores (HR and perceived exertion) and BOX VR scores. The results of the other qualitative variables, described individually, are shown below.

Table 2. Effort scores (HR and perceived exertion) and BOX VR scores for each session.

\begin{tabular}{ccccc}
\hline Exergaming Sessions & P1 & P2 & P3 & P4 \\
\hline Session 1 (training) & & & & \\
HR mean -bpm- & 90 & 105 & 115 & 108 \\
Borg score (TS) & $3 / 10$ & $5 / 10$ & $5 / 10$ & $5 / 10$ \\
BOX VR score & 1825 & 3613 & 3505 & 1967 \\
\hline Session 2 (training) & & & & \\
HR mean -bpm- & 86 & 110 & 125 & 118 \\
Borg score (TS) & $3 / 10$ & $7 / 10$ & $7 / 10$ & $8 / 10$ \\
BOX VR score & 1965 & 5441 & 6656 & 2361 \\
\hline
\end{tabular}

bpm: beats per minute; HR: heart rate; TS: training session.

\section{Participant 1}

The SSQ did not show cybersickness symptoms in either session. The SUS determined a very good usability $(100 / 100)$ in both experiences. High average values were recorded for positive experiences in the post-game GEQ (3.08/4), and low or zero values for negative experiences $(0.08 / 4)$. The satisfaction questionnaire reported positive experiences, describing 
it as being "motivating, de-stressing and useful for people of his age". He also considered "the virtual exercise to be useful", and commented that in the immersive environment he had not noticed the diplopia from which he suffers.

\section{Participant 2}

No cybersickness symptoms were reported in either session after training. The SUS was good (85/100 first session and 90/100 s session). The item "I think the device was easy to use" obtained the lowest score in the first session and the highest in the second. In the personal experiences post-game GEQ, participant 2 logged high average markers in positive experiences $(3.49 / 4)$ and low markers in negative experiences $(0.08 / 4)$ for both sessions. Qualitatively, he considered the experience to be "positive, relaxing and to be repeated", and demonstrated his preference for the exergaming session because "it motivates you to exercise and it is recommendable for older people".

\section{Participant 3}

SSQ showed no symptoms associated with cybersickness. Usability was rated with the highest score in the SUS (100/100). The values obtained in the post-game GEQ indicated that the experience was positive (3.41/4 in positive items and 0.08/4 in negative items), though participant 3 was notable in differing from the other participants, stating in item 17 that the feeling of having returned from a trip was moderate. In the satisfaction questionnaire, the characteristic that he liked the most was that the experience was "positive and that it transports you to another reality", also stating that it is "motivating, facilitates cognitive development and physical mobility" and that he would repeat the experience.

\section{Participant 4}

No symptoms associated with cybersickness were reported over the two sessions after completing the SSQ. The SUS showed that usability was good (85/100 and 95/100 in the first and second sessions, respectively), although he stated that he "would need someone's help to use the device". The experiences were considered good after the sessions, with high average scores on positive experiences (3.24/4) and low scores on negative experiences $(0.16 / 4)$ in the post-game GEQ. In the satisfaction questionnaire, he noted that "it was a very positive experience, being able to exercise as if you were in a gym, as well as improving your reflexes".

\section{Discussion}

Our outcomes support the feasibility of the HTC Vive Pro ${ }^{\mathrm{TM}}$ commercial HMD device as a tool to provide safe practice of physical exercise by healthy older people. In accordance with previous studies that have proposed guided and supervised sessions to facilitate senior populations' access to new technology [22,36], we consider it important to maintain these sessions, or at the very least to conduct initial training that will allow for future self-management at homes.

Furthermore, the answers on the usability and experience scales predict more learning and greater confidence in the handling of the tool the more it is used. We consider that this point, together with the absence of adverse symptoms, highlights the safety of the proposed games. Finally, adding a wireless technology to the HMD resulted in a major improvement to the safety of the immersive experiences and the comfort of the participants, as we have suggested in previous works [22].

We found high levels of user satisfaction which, as pointed out in other studies $[22,37,38]$, seems to position this virtual tool as an important element in the motivation of this group towards engaging in physical activity. According to Heredia et al. [39], the adherence to these exergaming programmes could help to make the physical and psychosocial benefits of exercise more accessible to the elderly, especially in the difficult times in which we are currently living.

To assess the suitability of the intended activities, the evaluation of both the perceived effort and HR during the immersive tasks was proposed. This point, which has already been 
raised in previous research with young adults (18-37 years) [40], has not yet been studied in older people. It could be of interest for possible incorporation into future protocols, not only as a control measure, but also to achieve a more individual, tailored design of the physical workload, or in its adaptation to possible existing disabilities. Even so, BOX VR seems to be a good option-an exergame that is quite close to the physical exercise workload in the real world, and without adverse effects (no cybersickness symptoms).

In line with the points outlined above, and as proposed by Donegan et al. [41], the suitability of the proposed exercises depends on a gradual increase in difficulty, with easy tasks comprising the first experiences, followed by more difficult tasks in the next. In the case of the present study, the second session was better accepted over all factors, with scores improving in the general indicators of usability, personal experiences, and satisfaction. Proof of this was also evident in an improved performance of the tasks, since $100 \%$ of the participants' scores improved when compared to their first attempts. This fact also led to an increase in the effort levels (mainly in the perceived effort), and confirms-as one of the participants stated: "a very positive experience, being able to exercise as if you were in a gym" - that the proposed exergaming could recreate virtually at home the possibilities and benefits of engaging in physical exercise/sporting activities in real environments.

Furthermore, the protocols for the current health emergency discourage group activities, meetings, and unnecessary movements of the population, especially for the groups most at risk from the virus. It is also fundamental to ensure that a safe social distance is observed. Nevertheless, it is also important to maintain a level of physical activity, and even more so to achieve healthy aging.

Furthermore, in line with the findings of recent publications [42], the use of this tool as an aid in these difficult times could represent a good opportunity to encourage sedentary people to establish healthy habits that endure over time.

Nevertheless, IVR also has its weaknesses. One of these is the cost of complex immersive VR devices, traditionally only available in research centres or specialised laboratories, and far beyond the reach of the general public. Fortunately, the availability of commercial HMDs has narrowed the gap, and these are now available for around EUR 500. This opens up the possibility of these devices being brought into the home environment [41].

Limitations

These preliminary outcomes are promising, but there are some limitations: One of them is that most of the variables are qualitative, and the fun and novel VR training may have favoured our findings. In addition, the low number of participants (without women), limited number of sessions, and similar VR environment may have impacted our results.

Therefore, we encourage future research with larger sample sizes, training protocols with several sessions per week and adapted to the target population, clinical trials with longer interventions, and a variety of virtual scenarios and proposed tasks. All of these improvements, when combined with the positive, entertaining, and useful nature of VR exercise as expressed by the participants, should help in the success of immersive virtual exercise programs and improve the roll-out of exergaming programmes focused on this population, as well as their possible future applications in their homes.

\section{Conclusions}

The IVR video games presented, based on commercial HMDs, are feasible in healthy older adults, and could motivate the practice of physical exercise in this population. In the cases reported, the sessions were safe (no adverse events), with positive opinions of the experience, very good usability, and high participant satisfaction.

The outcomes of this first approach at emulating motions based on best exercise practices recommended for older people provide motivation for future work. In addition, software, HMD, and IVR exergames that are commercially available were tested, and achieved enough exertion to be compared with conventional exercise activities. 
Exergaming is a viable alternative to traditional senior exercise, and could be a solution to the current situation arising from the impact of the COVID-19 pandemic. Nevertheless, further work is needed in this field to confirm its long-term feasibility.

Author Contributions: Conceptualization, methodology, and formal analysis: P.C.-P., G.R.-F. and J.M.C.-C. (all authors); software, validation, data curation, and visualization: P.C.-P.; resources, G.R.-F.; writing—original draft preparation, review, and editing: P.C.-P., G.R.-F. and J.M.C.-C. (all authors); supervision, project administration, and funding acquisition: P.C.-P. All authors have read and agreed to the published version of the manuscript.

Funding: Predoctoral fellowship of the Galician Government (ED481A-2019/158), Xunta de Galicia, Spain.

Institutional Review Board Statement: This study was conducted in accordance with the guidelines of the Declaration of Helsinki and approved by the Ethics Committee of Faculty of Physiotherapy, University of Vigo (protocol code 205-202-2 and date of approval 30 September 2020).

Informed Consent Statement: Informed consent was obtained from all subjects involved in the study.

Data Availability Statement: The data presented in this study are available on request from the corresponding author.

Conflicts of Interest: The authors declare no conflict of interest.

\section{References}

1. Zunzunegui, M.V.; Béland, F. Intersectorial policies to address the challenge of active aging. SESPAS report 2010. Gac. Sanit. 2010, 24, 68-73. [CrossRef]

2. INE Statistics. [Population Projections 2018-2068]. Statistics National Institute of Spain. 2018. Available online: https://ine.es / prensa/pp_2018_2068.pdf (accessed on 30 August 2021).

3. World Health Organization Active Ageing: A Policy Framework. 2002. Available online: https://apps.who.int/iris/handle/1066 5/67215 (accessed on 25 August 2021).

4. World Health Organization. World Report on Ageing and Health. 2015. Available online: https://apps.who.int/iris/handle/10 665/186463 (accessed on 25 August 2021).

5. Menéndez, C.; Brochier Kist, R.B. Physical activity and motor skills in the elderly: Their contributions to active, healthy and satisfactory aging. Textos Contextos (Porto Alegre) 2011, 10, 179-192.

6. Bherer, L.; Erickson, K.I.; Liu-Ambrose, T. A review of the effects of physical activity and exercise on cognitive and brain functions in older adults. J. Aging Res. 2013, 2013, 1-8. [CrossRef]

7. Chase, J.A.D.; Phillips, L.J.; Brown, M. Physical activity intervention effects on physical function among community-dwelling older adults: A systematic review and meta-analysis. J. Aging Phys. Act. 2017, 25, 149-170. [CrossRef] [PubMed]

8. Emery, C.F.; Hauck, E.R.; Blumenthal, J.A. Exercise adherence or maintenance among older adults: 1-year follow-up study. Psychol. Aging 1992, 7, 466-470. [CrossRef] [PubMed]

9. Rivera-Torres, S.; Fahey, T.D.; Rivera, M.A. Adherence to exercise programs in older adults: Informative report. Gerontol. Geriatr. Med. 2019, 5, 1-10. [CrossRef]

10. Picorelli, A.M.A.; Pereira, L.S.M.; Pereira, D.S.; Felício, D.; Sherrington, C. Adherence to exercise programs for older people is influenced by program characteristics and personal factors: A systematic review. J. Physiother. 2014, 60, 151-156. [CrossRef]

11. Lai, C.-C.; Shih, T.P.; Ko, W.C.; Tang, H.J.; Hsueh, P.R. Severe acute respiratory syndrome coronavirus 2 (SARS-CoV-2) and coronavirus disease-2019 (COVID-19): The epidemic and the challenges. Int. J. Antimicrob. Agents 2020, 55, 105924. [CrossRef] [PubMed]

12. Helmich, R.C.; Bloem, B.R. The impact of the COVID-19 pandemic on parkinson's disease: Hidden sorrows and emerging opportunities. J. Park Dis. 2020, 10, 351-354. [CrossRef]

13. Prem, K.; Liu, Y.; Russell, T.W.; Kucharski, A.J.; Eggo, R.M.; Davies, N. The effect of control strategies to reduce social mixing on outcomes of the COVID-19 epidemic in Wuhan, China: A modelling study. Lancet Public Health 2020, 5, E261-E270. [CrossRef]

14. Wong, A.Y.Y.; Ling, S.K.K.; Louie, L.H.T.; Law, G.Y.K.; So, R.C.H.; Lee, D.C.W.; Yau, F.C.F.; Yung, P.S.H. Impact of the COVID-19 pandemic on sports and exercise. Asia-Pac. J. Sports Med. Arthrosc. Rehabil. Technol. 2020, 22, 39-44. [CrossRef]

15. Mutz, M.; Gerke, M. Sport and exercise in times of self-quarantine: How germans changed their behaviour at the beginning of the covid-19 pandemic. Int. Rev. Sociol. Sport 2021, 56, 305-316. [CrossRef]

16. Rodríguez, M.A.; Crespo, I.; Olmedillas, H. Exercising in times of COVID-19: What do experts recommend doing within four walls? Rev. Esp. Cardiol. 2020, 73, 527-529. [CrossRef]

17. Yang, Y.C.; Chou, C.L.; Kao, C.L. Exercise, nutrition, and medication considerations in the light of the COVID pandemic, with specific focus on geriatric population: A literature review. J. Chin. Med. Assoc. 2020, 83, 977-980. [CrossRef] [PubMed] 
18. Graves, L.E.F.; Ridgers, N.D.; Williams, K.; Stratton, G.; Atkinson, G.; Cable, N.T. The physiological cost and enjoyment of wii fit in adolescents, young adults, and older adults. J. Phys. Act. Health 2010, 7, 393-401. [CrossRef] [PubMed]

19. Viana, R.B.; Vancini, R.L.; Vieira, C.A.; Gentil, P.; Campos, M.H.; Andrade, M.S.; de Lira, C.A.B. Profiling exercise intensity during the exergame hollywood workout on XBOX 360 kinect ${ }^{\circledR}$. Peer] 2018, 6, e5574. [CrossRef] [PubMed]

20. Viana, R.B.; de Lira, C.A.B. Exergames as coping strategies for anxiety disorders during the COVID-19 quarantine period. Games Health J. 2020, 9, 147-149. [CrossRef]

21. Ambrosino, P.; Fuschillo, S.; Papa, A.; Minno, M.N.D.D.; Maniscalco, M. Exergaming as a Supportive Tool for Home-Based Rehabilitation in the COVID-19 Pandemic Era. Games Health J. 2020, 9, 311-313. [CrossRef] [PubMed]

22. Campo-Prieto, P.; Carral, J.M.C.; Oliveira, I.; Rodríguez-Fuentes, G. Immersive virtual reality in older people: A case study. Retos 2021, 39, 1001-1005. [CrossRef]

23. Davis, S.; Nesbitt, K.; Nalivaiko, E. A systematic review of cybersickness. In Proceedings of the 2014 Conference on Interactive Entertainment-IE2014, Newcastle, NSW, Australia, 2-3 December 2014; ACM Press: New York, NY, USA, 2014; pp. 1-9. [CrossRef]

24. Campo-Prieto, P.; Cancela, J.M.C.; Rodríguez-Fuentes, G. Immersive virtual reality as physical therapy in older adults: Present or future (systematic review). Virtual Real. 2021, 25, 1-17. [CrossRef]

25. Kim, A.; Darakjian, N.; Finley, J.M. Walking in fully immersive virtual environments: An evaluation of potential adverse effects in older adults and individuals with Parkinson's disease. J. Neuroeng. Rehabil. 2017, 14, 16. [CrossRef] [PubMed]

26. Jung, J.; Yu, J.; Kang, H. Effects of virtual reality treadmill training on balance and balance self-efficacy in stroke patients with a history of falling. J. Phys. Ther. Sci. 2012, 24, 1133-1136. [CrossRef]

27. Kennedy, R.S.; Lane, N.E.; Berbaum, K.S.; Lilienthal, M.G. Simulator sickness questionnaire: An enhanced method for quantifying simulator sickness. Int. J. Aviat Psychol. 1993, 3, 203-220. [CrossRef]

28. Kennedy, R.S.; Drexler, J.; Kennedy, R.C. Research in visually induced motion sickness. Appl. Ergon. 2010, 41, 494-503. [CrossRef]

29. Lawson, B.D.; Graeber, D.A.; Mead, A.M.; Muth, E.R. Signs and symptoms of human syndromes associated with synthetic experiences. In Handbook of Virtual Environments: Design, Implementation, and Applications; Hale, K.S., Stanney, K.M., Eds.; Lawrence Erlbaum Associates Publishers: Mahwah, NJ, USA, 2002; pp. 589-618.

30. Brooke, J. SUS: A quick and dirty usability scale. Usability Eval. Ind. 1995, 189, 4-7. [CrossRef]

31. Aguilar, M.I.H.; Villegas, A.A.G. Comparative analysis of the system usability scale (SUS) in two versions. Rev. Iberoam. Las Cienc. Comput. Inf. 2016, 5, 44-58.

32. Ijsselsteijn, W.A.; de Kort, Y.A.W.; Poels, K. The Game Experience Questionnaire; Eindh Tech Univ Eindh: Eindhoven, The Netherlands, 2013.

33. Denisova, A.; Nordin, A.I.; Cairns, P. The convergence of player experience questionnaires. In Proceedings of the 2016 Annual Symposium on Computer-Human Interaction in Play CHI PLAY 16, Austin, TX, USA, 16-19 October 2016; Association for Computing Machinery: New York, NY, USA, 2016; pp. 33-37. [CrossRef]

34. Borg, G.A.V. Psychophysical bases of perceived exertion. Med. Sci. Sports Exerc. 1982, 14, 377-381.

35. Bank, P.J.M.; Cidota, M.A.; Ouwehand, P.E.W.; Lukosch, S.G. Patient-tailored augmented reality games for assessing upper extremity motor impairments in parkinson's disease and stroke. J. Med. Syst. 2018, 42, 246. [CrossRef] [PubMed]

36. Roberts, A.R.; Schutter, B.D.; Franks, K.; Radina, M.E. Older adults' experiences with audiovisual virtual reality: Perceived usefulness and other factors influencing technology acceptance. Clin. Gerontol. 2019, 42, 27-33. [CrossRef] [PubMed]

37. Duque, G.; Boersma, D.; Loza-Diaz, G.; Hassan, S.; Suarez, H.; Geisinger, D.; Suriyaarachchi, P.; Sharma, A.; Demontiero, O. Effects of balance training using a virtual-reality system in older fallers. Clin. Interv. Aging. 2013, 8, 257-263. [CrossRef] [PubMed]

38. Benham, S.; Kang, M.; Grampurohit, N. Immersive virtual reality for the management of main in mommunity-dwelling older adults. OTJR Occup. Particip. Health 2019, 39, 90-96. [CrossRef] [PubMed]

39. Heredia, N.M.; Rodríguez, E.S.; Rodríguez-García, A.M. Benefits of physical activity for promoting active aging in older people.Bibliographic review. Retos 2020, 39, 829-834. [CrossRef]

40. Yoo, S.; Ackad, C.; Heywood, T.; Kay, J. Evaluating the actual and perceived exertion provided by virtual reality games. In Proceedings of the 2017 CHI Conference Extended Abstracts on Human Factors in Computing Systems CHI PLAY 17, Amsterdam, The Netherlands, 15-18 October 2017; Association for Computing Machinery: New York, NY, USA; pp. 3050-3057.

41. Donegan, T.; Ryan, B.E.; Swidrak, J.; Sanchez-Vives, M.V. Immersive virtual reality for clinical pain: Considerations for effective therapy. Front Virtual Real. 2020, 1, 9. [CrossRef]

42. Rodríguez, M.A.; Fernández, H.O. COVID-19: A challenge for exercisers and, an opportunity for not-exercisers? Arch Med. Deporte. 2020, 37, 150-151. 